\title{
Clinical Scoring System to Predict Malarial Fever: A Prospective Study
}

\author{
Viral shah*, B.K.Shah ${ }^{\dagger}$, Bhavin Vadera ${ }^{\ddagger}$, H.K.Acharya ${ }^{\S}$ \\ ${ }^{*}$ Department of Medicine, Medical College, Bhavnagar, Gujarat, India. ${ }^{\dagger}$ Department of Pharmacology, M.P. Shah Medical College, Jamnagar, \\ Gujarat, India. ₹Department of P\&SM, M.P. Shah Medical College, Jamnagar, Gujarat, India. \\ ${ }^{\S}$ Department of Medicine, M.P. Shah Medical College, Jamnagar, Gujarat, India.
}

\section{A B STRACT}

Background: Malaria is the most important infectious disease of mankind. Though, smear examination is gold standard in diagnosing malaria, it has many drawbacks particularly in developing countries. Methods: A prospective study was designed with duration of the study was six months. Based on previous work, we identified the most specific clinical features of malaria and assigned with score. Maximum score was 14. Only adult patients with more than 13 years of age with complaint of fever were included for the study. Clinical Score was calculated for each patient by two different persons (clinician and intern). All the patients were subjected for peripheral smear by specialist or trained person to diagnose malaria. Clinical Score were compared with smear diagnosis of malaria. Sensitivity, specificity, positive predictive value (PPV) and negative predictive values (NPV) for each score were analyzed. Receiver Operative Curve (ROC) was prepared to assign a particular score which can diagnose malaria with good certainty. Results: A total of 142 consecutive fever cases were included in the study. 67 (47.18\%) cases were of malaria and rests were control cases. Using score of more than eight, the sensitivity, specificity, positive predictive value and negative predictive value of clinical scoring system to detect malaria (either vivax or falciparum) were $95.52 \%, 85.33 \%, 85.33 \%$ and $95.52 \%$ respectively. Using score of more than nine, the same statistics were $91.04 \%, 93.33 \%, 92.42 \%$ and $92.10 \%$ respectively. Using ROC curve score more than or equal to nine can give the clinical diagnosis of malaria with good certainty. Conclusion: Score more than nine can be used in clinics to assign as malaria with good confidence and can be treated empirically with antimalarials. This score can substitutes smear examination however, can not replace it. This scoring system is very helpful where smear examination is remote possibility or it can not be relied upon due to technical or staff problem specifically in rural part of India or peripheral health centre with least facilities.

Key words: Peripheral smear examination, malaria, clinical score system.

\section{INTRODUCTION}

Malaria is most important parasitic disease of human being affecting over $40 \%$ of the world population, ${ }^{1}$ and causing about 1 million deaths. Though there has been decline in the total number of reported cases from India, the P. falciparum cases have registered significant increase. ${ }^{2}$ Malaria has protean manifestations varied from simple flu like illness to rapidly fatal cerebral malaria. The main diagnostic tool for malaria is peripheral smear examination. ${ }^{3}$ There are numerous fallacies which makes it unreliable. Few of them are; lack of trained persons, failure to recognize or misclassify the parasite by technicians, work load in government

Address for correspondence:

Phone: 09872308785

Email: viralshah_ratonal@yahoo.co.in

DOI: 10.5530/ijmedph.2.2011.7 hospitals, prior antimalarial drugs etc. ${ }^{3,45}$ With the availability of Rapid Diagnostic test using HRPf or LDH antigen (RDT), problem was thought to be overcome. ${ }^{6,7,8}$ However, the sensitivity of RDT can not be comparable to that of smear and cost and non availability in many rural area of India makes it problematic. ${ }^{7,8,9}$ Over treatment with antimalarial drugs in nonmalarial acute undifferentiated fever is the main problem in rural India. ${ }^{10}$ Rampant use of antimalarials may pose problems like side effects of drugs, drug resistance and cost. Considering these issues in mind, a clinical scoring system was devised, aimed to predict the diagnosis of malaria with fair confidence and make a dent in these burning problems.

\section{Methods \\ Patient selection}

The study duration was of 6 months (from April to September 2008). This period was chosen because maximum incidence of malaria seen around this time period. 
Study was conducted at Sir T General Hospital \& Government Medical College, Bhavnagar, Gujarat, India. Ethical Committee permission was taken. The study was approved by the committee.

Total 142 patients presented with the complaints of fever irrespective of duration and characteristic were included in the study. All these patients were selected non-priority random selection. This total includes indoor as well as outdoor patients.

Inclusion criteria: 1) Patients were adult more than 13 years of age. 2) Fever as a presenting complaints irrespective of duration and characteristic.

Exclusion criteria: 1) Pediatric patients (less than 13 years) were excluded. 2) One patient with mixed infection was also excluded because it was the single patient and hence, statistical correlation is difficult. 3) Undiagnosed cases and pyrexia of unknown origin cases were excluded from the study.

\section{Clinical Scoring system}

Based on our previous (unpublished) work, the specific features of malaria were noted and assigned with score. The scoring devise is presented in Table-1. Maximum score was 14 points. In every patient the clinical score was calculated. Score was calculated by the investigator (VNS) and internist independently. Higher score was taken in to account whenever there was dichotomy.

\section{Study protocol and design}

Malaria was diagnosed by peripheral smear examination by trained technician or pathologist. HRPf 2 kits were used in emergency for the diagnosis of malaria and also confirmed by smear examination later on. All the patients

\begin{tabular}{|c|c|}
\hline \multicolumn{2}{|c|}{ Positive Scores } \\
\hline Season/epidemic period ${ }^{\#}$ & +3 points \\
\hline Alternative diagnosis less likely* & +3 points \\
\hline Fever & +2 points \\
\hline Non pulsatile headache & +2 points \\
\hline Chills \& rigor & +2 points \\
\hline Intermittent nature & +2 points \\
\hline \multicolumn{2}{|c|}{ Negative Scores } \\
\hline Conjunctival suffusion & -1 point \\
\hline Rash & -1 point \\
\hline Respiratory Rate >28 & -1 point \\
\hline Sever arthralgia/myalgia & -1 point \\
\hline Throat congestion & -1 point \\
\hline Fever $>40$ Celsius & -1 point \\
\hline Continuous fever & -1 point \\
\hline
\end{tabular}

were investigated thoroughly for febrile illness with appropriate investigations. Diagnosis was recorded separately by investigator. Those patients with the diagnosis of malaria either P.vivax, P.falciparum or mixed were noted and their scores were compared with the other patients who were negative for malaria.

\section{Statistical analysis}

Categorical data presented as percentage. Sensitivity, specificity, positive predictive value (PPV) and negative predictive values (NPV) were calculated using standard statistical formula using SPSS 10 software. Receiver Operative Curve (ROC) was derived to select the clinical score which can be most predictive. $p$ value of less than 0.05 was taken as statistical significant.

\section{RESULTS}

One hundred and forty two cases were studied. All the cases were presented with the complaint of fever irrespective of duration and or characteristic. Eighty were males (56.33\%) and rest females (43.67\%). Sixty seven $(47.18 \%)$ were diagnosed as malaria and $75(52.82 \%)$ were other febrile cases. Mean age \pm SD of patients were $34 \pm 15$ years. The distribution of malarial and other febrile cases is given in table no. 2.

From 67 malarial cases, 26 were Plasmodium vivax (P.vivax) malaria $(38.80 \%)$ and 41 Plasmodium falciparum (P.falciparum) malaria $(61.19 \%)$. Most common age group affected with malaria either falciparum or vivax was $21-30$ years $(74.62 \%)$.

Clinical score was calculated for all the patients as described above. Average score of all patients was 8.83. Mean clinical score for malarial patients was 11.58 and of non-malarial

\begin{tabular}{lcc}
$\begin{array}{l}\text { Table 2: Distribution of cases of fever with clinical } \\
\text { scores }\end{array}$ & $\begin{array}{c}\text { No of } \\
\text { patients }\end{array}$ & $\begin{array}{c}\text { Average score } \\
\text { (range) }\end{array}$ \\
\hline \multicolumn{1}{c}{ Diagnosis } & 26 & $11.15(8-14)$ \\
\hline P. vivax malaria & 41 & $11.85(7-14)$ \\
P. falciparum malaria & 67 & 11.58 \\
Malaria & 8 & $6.25(3-8)$ \\
Enteric fever & 19 & $7.16(6-10)$ \\
Viral fever & 9 & $6.56(2-11)$ \\
Upper respiratory tract infection & 12 & $5.42(2-11)$ \\
Pneumonia & 6 & $6.42(5-8)$ \\
Tuberculosis & 9 & $6.33(1-10)$ \\
Hepatitis & 1 & 7 \\
Meningitis & 7 & $4.57(1-8)$ \\
Bacterial pleural effusion & 2 & $8.5(8-9)$ \\
HIV & 2 & 8 \\
UTI & 75 & 6.37 \\
Non-malaria & 142 & $\mathbf{8 . 8 3}$ \\
Total & & \\
\hline
\end{tabular}




\begin{tabular}{|c|c|c|c|c|}
\hline Criterion & Sensitivity & $95 \% \mathrm{Cl}$ & Specificity & $95 \% \mathrm{Cl}$ \\
\hline$>=1$ & 100.00 & $94.6-100.0$ & 0.00 & $0.0-4.8$ \\
\hline$>1$ & 100.00 & $94.6-100.0$ & 2.67 & $0.4-9.3$ \\
\hline$>2$ & 100.00 & $94.6-100.0$ & 5.33 & $1.5-13.1$ \\
\hline$>3$ & 100.00 & $94.6-100.0$ & 12.00 & $5.6-21.6$ \\
\hline$>4$ & 100.00 & $94.6-100.0$ & 17.33 & $9.6-27.8$ \\
\hline$>5$ & 100.00 & $94.6-100.0$ & 30.67 & $20.5-42.4$ \\
\hline$>6$ & 100.00 & $94.6-100.0$ & 49.33 & $37.6-61.1$ \\
\hline$>7$ & 97.01 & 89.6-99.6 & 69.33 & $57.6-79.5$ \\
\hline$>8$ & 95.52 & $87.5-99.0$ & 85.33 & $75.3-92.4$ \\
\hline$>9$ & 91.04 & $81.5-96.6$ & 93.33 & $85.1-97.8$ \\
\hline$>10$ & 74.63 & $62.5-84.5$ & 97.33 & $90.7-99.6$ \\
\hline$>11$ & 53.73 & $41.1-66.0$ & 100.00 & $95.2-100.0$ \\
\hline$>12$ & 35.82 & $24.5-48.5$ & 100.00 & $95.2-100.0$ \\
\hline$>13$ & 10.45 & $4.3-20.4$ & 100.00 & $95.2-100.0$ \\
\hline$>14$ & 0.00 & $0.0-5.4$ & 100.00 & $95.2-100.0$ \\
\hline
\end{tabular}

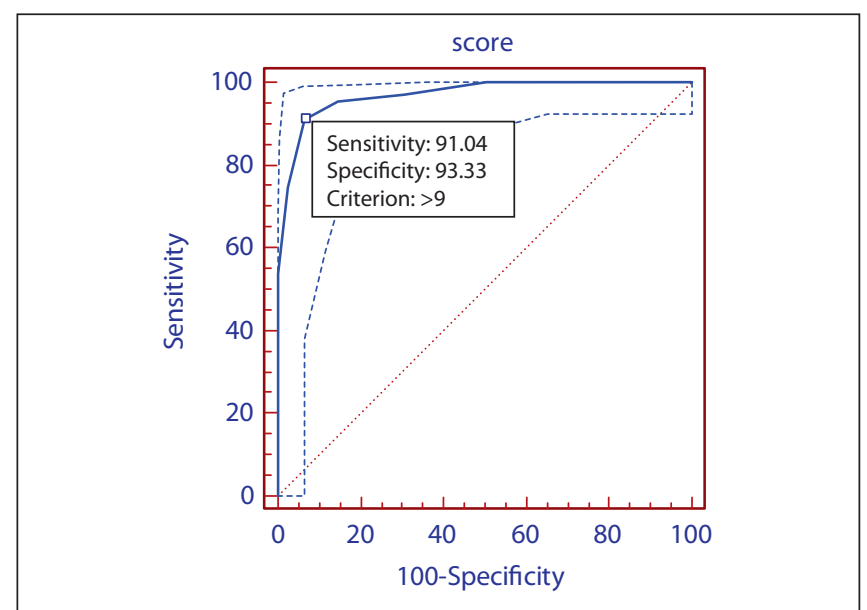

Figure 1: ROC curve for different clinical score for the predicting malaria

patients was 6.37. Detailed scoring of all the cases is depicted in table no. 2. Sensitivity and specificity at different cut off clinical score are shown in Table 3.

At the cut off value of more than nine; sensitivity of this scoring system to detect malaria (either falciparum or vivex) was $91.04 \%$, specificity was $93.33 \%$, PPV was $92.42 \%$ and NPV was $92.10 \%$. The score would detect falciparum malaria with $95.12 \%$ sensitivity, $93.33 \%$ specificity, $86.63 \%$ PPV and $97.22 \%$ NPV. Sensitivity, specificity, PPV and NPV to detect vivex was $84.61 \%, 93.33 \%, 81.48 \%$ and $94.59 \%$ respectively.

The cut off value where optimum sensitivity and specificity can be obtained was more than nine. With the help of receiver operating curve (ROC) analysis cut off value of clinical scoring was decided. At score value of more than 9, area under curve (AUC) was 0.97 (95\% CI 0.93-0.99) which was statistically significant $(p<0.001)$ which is illustrated in figure 1.

\section{DISCUSSION}

This is the first prospective study from India to derive a clinical score system to diagnose malaria clinically.

The main diagnostic tool for the malaria is peripheral smear examination for the malarial parasite. However, this test is not the panacea in diagnosis of malaria due to many limitations of the test. As stated in introduction, there are numerous fallacies resulting in the misdiagnosis of malaria. Misdiagnosis of malaria by peripheral examination is recognized as a cause for under reporting by few studies. ${ }^{11,12}$

RDT is has its own problem in developing countries like India. ${ }^{6,789}$ The use of empirical antimalarials by clinicians was rampant ${ }^{10}$ in rural part of country due to difficulty in diagnosis of malaria.

The conclusion by Doherty ${ }^{13}$ et al. "high index of suspicion is the main diagnostic tool in malaria" still holds true.

Use of empirical antimalarials is rampant in India ${ }^{10}$ particularly at primary health centers due to difficulties in diagnosing malaria in adverse situations as mentioned above. Empirical use is not justified as it may results in to over use of drugs, side effects associated with drugs, development of resistant parasite and cost. Therefore, the clinical scoring was of prime importance particularly in the area where facilities were sparse, lack of availability of trained staffs.

As describe in results of the present study, using score of more than nine gives good sensitivity $(91.04 \%)$, and specificity $(93.33 \%)$.

It can be used clinically to increase suspicion or for empirical treatment of malaria when microscopy is not feasible or 
can not be relied upon. Area under ROC curve was 0.97 using the scoring system prepared in the study.

Limitations of our studies were 1) As only adult patients were included, this should not be applied to pediatric patients. 2) The smear is gold standard when examined by experts and hence, this score system should be viewed as helping tool for physicians working in area where availability of smear or its validity is uncertain. 3) Large trial is needed to prove its effectiveness in primary care setting.

Nevertheless, this study may be helpful in epidemics or developing countries where antimalarial drugs are being used empirically. Though, study population is small; finding of this study is not ignorable.

\section{ACKNOWLEDGEMENT}

We acknowledge the help rendered by Dr. chandrika and Dr. Khushbu (internists) for calculating the clinical score, Dr. Deval Rajyaguru and Dr. Krunal for their help in collecting data. The help by Dr. B.D. Parmar, Dean, medical college Bhavnagar and Dr. C.B.Tripathi is also worth mentioning.

\section{REFERENCES}

1. Nosten F, Price RN. Newer antimalarials, a risk benefit analysis. Drug Saf 1995; 112:264-73.

2. Sharma VP. Reemergence of malaria in India. Ind J Med Res 1996; 103:26-45.
3. Payne D. Use and limitations of light microscopy for diagnosing malaria at the primary health care. Bull WHO. 1988; 66:621-626.

4. Durrheim DN, Becker PJ, Billinghurst K. Diagnostic disagreement - the lessons learnt from malaria diagnosis in Mpumalanga. S Afr Med J 1997; 87:1016.

5. Shanker Matta, S.L. Kantharia, V.K. Desai. Malaria diagnosis in private laboratories of Surat city: a laboratory based study. J Vect Borne Dis 2004; 41:76-79.

6. Shiff $C J$, Premji Z, Minjas JN. The rapid Parasight $F$ test. A new diagnostic tool for plasmodium falciparum infection. Trans R Soc Soc Med Hyg 1993; 87:29-31.

7. Mharakurwa $\mathrm{B}$ et al. Trial of the Parasight $\mathrm{F}$ test for the malaria diagnosis in the primary health care system, Zimbabwe. Trop Med and International Health 1997; 2:544-550.

8. Craig MH, Sharp BL. Comparative evaluation of four techniques for the diagnosis of plasmodium falciparum malaria. Trans $R$ Soc Soc Med Hyg 1997; 91:279-282.

9. Trarore I etal. Field studies of the Parasight $F$ test in a malaria endemic area: cost, feasibility, sensitivity, specificity, predictive value and detection of the HRP 2 gene among wild type plasmodium falciparum in Mali. Am J Trop Med Hyg 1998.

10. Joshi R, Colford JM Jr, Reingold AL, Kalantri S. Nonmalarial acute undifferentiated fever in a rural hospital in central India: diagnostic uncertainty and overtreatment with antimalarial agents. Am J Trop Med Hyg. 2008; 78:393-9.

11. Dev V, Sharma VP,. Persistent transmission of malaria in Sonapur PHC, Kamrup district, Assam. J Parasitic Dis 1995; 19:65-68.

12. Srivastava HC, Kant R, Bhatt RM, Sharma SK, Sharma VP. Epidemiological observations on malaria in villages of Buhari PHC, Surat, Gujarat. Indian J Malariol 1995; 32:140-152.

13. Doherty JF, Grant AD, Bryceson ADM. Fever as presenting complaint of travelers returning from tropics. Q J Med 1995; 88:277-81.

14. Breman JG,. The ears of the hippopotamus: manifestations, determinants and estimation of the malaria burden. Am J Trop Med Hyg 2001; 64 (Suppl 1):1-11.

15. Snow RW, Guerra CA, Noor AM, Myint HY, Hay SI, The global distribution of clinical episodes of Plasmodium falci-parum malaria. Nature 2005; 434:214-217. 